\title{
Obesity and the Link to Chronic Disease Development
}

\author{
Reza Hakkak ${ }^{1-3^{*}}$ and Andrea Bell ${ }^{1,3}$ \\ ${ }^{1}$ Department of Dietetics and Nutrition, University of Arkansas for Medical Sciences, Little Rock, AR 72205, USA \\ ${ }^{2}$ Department of Pediatrics, University of Arkansas for Medical Sciences, Little Rock, AR 72205, USA \\ ${ }^{3}$ Arkansas Children's Research Institute, Little Rock, AR 72202, USA
}

Correspondence to:

Dr. Reza Hakkak

Department of Dietetics and Nutrition

4301 West Markham St., Mail Slot 627

Little Rock, AR 72205, USA

Tel: 501-686-6166

Fax: 501-686-5716

E-mail: HakkakReza@uams.edu

Received: October 12, 2016

Accepted: October 14, 2016

Published: October 14, 2016

Citation: Hakkak R, Bell A. 2016. Obesity and the Link to Chronic Disease Development.J Obes Chronic Dis 1(1): 1-3.

Copyright: C 2016 Hakkak and Bell. This is an Open Access article distributed under the terms of the Creative Commons Attribution 4.0 International License (CC-BY) (http:// creativecommons.org/licenses/by/4.0/) which permits commercial use, including reproduction, adaptation, and distribution of the article provided the original author and source are credited.

Published by United Scientific Group

\section{Introduction}

In the US, childhood obesity affects approximately 12.5 million children and teens. Changes in obesity prevalence show a rapid increase from the 1960 s to the 1980s and 1990s when obesity prevalence among children and teens tripled [1]. Recently, a nationally representative study of children and adolescents in the US, ages 2 to 19 years, showed that the prevalence of obesity from 2011 to 2014 was $17.0 \%$ and extreme obesity prevalence was $5.8 \%$ [2]. Childhood obesity can also increase risk factors for cardiovascular hypertension, high cholesterol, and diabetes.

Obesity has been an epidemic in the US for more than three decades, and the proportion of overweight and obese adults in the population continues to grow. On average, an adult in the US now weighs 24 pounds more than an adult in 1960 [3]. According to a recent data survey of adults in the US, the age-adjusted prevalence of obesity from 2013 to 2014 was $35.0 \%$ among men and $40.4 \%$ among women [4]. Similarly, many other countries are experiencing dramatic increases in obesity. Worldwide, greater than 1.9 billion adults are overweight-of which 600 million are obese [5]. These statistics have serious health implications due to the association between obesity and the risk for chronic diseases including certain types of cancer, type 2 diabetes, cardiovascular disease, and liver disease [6].

\section{Obesity and Cancer}

According to the American Cancer Society, cancer is the second leading cause of mortality in the US. It is estimated that 1,685,210 men and women in the US will be diagnosed with cancer in 2016 [7]. In men, prostate, lung, and colorectal cancers are most prevalent, and in women the most prevalent cancer types include lung, breast, and colorectal [7]. In 2016, it is estimated that 588,430 men and women will likely die from this disease [7].

According to the 2014 World Cancer Report by the International Agency for Research on Cancer, cancer is among the leading causes of morbidity and mortality worldwide. Approximately 14 million new cases of cancer and 8.2 million cancer-related deaths occurred worldwide in 2012 [8], and the number of new cases is expected to rise by approximately $70 \%$ over the next two decades. In 2012, the most common types of cancer in men worldwide were lung, prostate, colorectal, stomach, and liver cancer; among women, the five most common types were breast, colorectal, lung, cervix, and stomach cancer [8].

The role of obesity in carcinogenesis has been investigated for the past several years. Obesity is not only associated with increased incidence but also with increased mortality from various types of cancer $[8,9]$. Obesity is also linked with an increased risk of developing several types of cancers including cancers of the esophagus, pancreas, colon, rectum, breast, endometrium, kidney, thyroid, and 
gallbladder. According to the 2014 Cancer Progress Report from the American Association for Cancer Research, nearly $25 \%$ of the relative contribution to cancer incidence can be attributed to overweight/obesity-second only to tobacco use. Furthermore, when obesity is combined with lack of physical activity and poor diet, the relative contribution of newly diagnosed cancer cases in the US rises to $33 \%$ [9]. It is projected that the current increase in obesity rates could lead to about 500,000 additional cases of cancer in the US by 2030 [9].

According to the National Cancer Institute, several possible mechanisms have been suggested to explain the association between obesity and increased risk of developing certain types of cancers. 1) Fat tissue produces excess amounts of estrogen - high levels of which have been associated with the risk of breast, endometrial, and some other cancers. 2) Obesity can cause increased levels of insulin and insulin-like growth factor-1, which may promote the development of certain tumors. 3) Fat cells produce adipokines that may stimulate or inhibit cell growth. 4) Fat cells may also have direct and indirect effects on other tumor growth regulators including mammalian target of rapamycin (mTOR) and AMP-activated protein kinase. 5) Obese people often have chronic low-level (or "subacute") inflammation, which has been associated with an increased cancer risk [9]. Other possible mechanisms include altered immune responses, effects on the nuclear factor kappa beta system, and oxidative stress [9].

\section{Obesity and Type 2 Diabetes}

The prevalence of type 2 diabetes (T2D) has more than doubled worldwide in the past 20 years [10], and $80 \%$ of people with T2D are also obese [11]. Obesity causes adipocytes to be enlarged, which leads to the dysregulation of adipokine secretion [12]. The dysregulation of adipokines is thought to contribute to adipocyte inflammation and ultimately insulin resistance [12]. Insulin-resistant adipocytes can trigger, the release of free fatty acids from adipocytes leading to increased levels of circulating free fatty acids in the blood stream and deposition of fatty acids into the liver and muscle [12]. Elevated serum and intracellular lipids intensify insulin resistance by interfering with insulin signaling [13]. Insulin resistance occurring in the liver can further increase blood glucose levels as the liver will begin releasing glucose into the blood stream [13]. Thus, blood glucose levels remain high and the pancreas increases insulin secretion to attempt to rectify the high blood glucose [13]. The increased workload on the pancreas may damage pancreatic $\beta$ cells in the long-term and impair their ability to secrete insulin, which further challenges the body's ability to control blood glucose levels [13]. Over time, uncontrolled blood glucose can lead to complications such as kidney disease, neuropathy, infection/amputations, retinopathy, gastroparesis, diabetic ketoacidosis, high blood pressure, and stroke [14].

\section{Obesity and Cardiovascular Disease}

Worldwide, cardiovascular disease is the leading cause of death [15] and encompasses vascular and structural dysfunctions including coronary artery disease, heart failure, atrial fibrillation, and ischemic and hemorrhagic stroke. Obesity-associated adipocyte enlargement, systemic inflammation, and insulin resistance significantly contribute to the development of cardiovascular disease by increasing the risk of developing comorbidities such as hypertension, dyslipidemia, and T2D [16]. Obesity increases coronary artery disease risk by promoting atherosclerosis through obesityassociated inflammatory mechanisms [16]. The details behind obesity's association with increased risk for heart failure and atrial fibrillation are complex but are essentially, in part, due to obesity-associated high blood pressure, increased blood volume, and changes in adipokine secretion, as well as actual structural or blood flow changes related to increased body mass [16].

\section{Obesity and Liver Disease}

Obesity, T2D, and insulin resistance are independently associated with increased fat accumulation in the liver known as steatosis [6]. Steatosis initiates the development of nonalcoholic fatty liver disease (NAFLD), and as NAFLD progresses, hepatic inflammation (steatohepatitis) and cirrhosis can occur [6]. According to estimates, $30 \%$ to $40 \%$ of the US adult population has NAFLD [17]. Obesity increases the risk of developing NAFLD by 3.5 fold [18]. Metabolic complications of obesity play a significant role in the development of NAFLD [12]. The primary metabolic complications of NAFLD include inflammation, adipokine dysregulation, dyslipidemia, and insulin resistance [12]. As previously explained, obesityinduced adipocyte enlargement dysregulates secretion of leptin and adiponectin and causes accumulation of pro-inflammatory macrophages in adipose tissue leading to increased secretion of pro-inflammatory cytokines. This adipokine and cytokine dysregulation plays a role in initiating insulin resistance [12]. Because insulin signaling promotes the storage of lipids, an insulin resistant state causes adipose tissue to release free fatty acids into the blood [12]. When obesity is concentrated viscerally, free fatty acids enter the portal circulation and are deposited in the liver initiating liver steatosis [12].

\section{Summary}

Because obesity is a major risk factor for developing the aforementioned chronic diseases, maintaining a normal body weight, or losing weight, may be essential to reduce the risk of developing these diseases. Research has shown nutrition and lifestyle habits are fundamental to both weight maintenance and weight loss, but we also know that the resolution to the obesity epidemic will go beyond weight loss. By launching the new Journal of Obesity and Chronic Disease and with help from researchers and health care professionals, we can increase the understanding of all aspects of obesity as well as obesity's association with chronic diseases.

\section{Conflict of Interest Statement}

The authors declare that they have no conflict of interests. 


\section{Acknowledgments}

The authors wish to thank John Gregan for his valuable assistance in preparation of this manuscript.

\section{References}

1. Ogden CL, Carroll MD, Curtin LR, Lamb MM, Flegal KM. 2010. Prevalence of high body mass index in US children and adolescents, 2007-2008. JAMA 303(3): 242-249. doi: 10.1001/jama.2009.2012

2. Ogden CL, Carroll MD, Lawman HG, Fryar CD, Kruszon-Moran D, et al. 2016. Trends in obesity prevalence among children and adolescents in the United States, 1988-1994 through 2013-2014. JAMA 315(21): 2292-2299. doi: 10.1001/jama.2016.6361

3. Ogden CL, Fryar CD, Carroll MD, Flegal KM. 2004. Mean body weight, height, and body mass index, United States 1960-2002. Adv Data 347:1-17.

4. Flegal KM, Kruszon-Moran D, Carroll MD, Fryar CD, Ogden CL. 2016. Trends in obesity among adults in the United States, 2005 to 2014. JAMA 315(21): 2284-2291. doi: 10.1001/jama.2016.6458

5. Overweight \& Obesity. [http://www.cdc.gov/obesity/index.html]

6. Lazo M, Hernaez R, Eberhardt MS, Bonekamp S, Kamel I, et al. 2013. Prevalence of nonalcoholic fatty liver disease in the United States: the Third National Health and Nutrition Examination Survey, 1988-1994. Am J Epidemiol 178(1): 38-45. doi: 10.1093/aje/kws448

7. Cancer Facts and Figures 2016. [http://www.cancer.org/research/ cancerfactsstatistics/cancerfactsfigures2016]

8. Cancer Fact Sheets. [http://globocan.iarc.fr/Pages/fact_sheets_cancer. aspx]

9. Obesity and Cancer Risk. [https://www.cancer.gov/about-cancer/ causes-prevention/risk/obesity/obesity-fact-sheet]

10. Zimmet P, Alberti KG, Magliano DJ, Bennett PH. 2016. Diabetes mellitus statistics on prevalence and mortality: facts and fallacies. Nat Rev Endocrinol 12(10): 616-622. doi: 10.1038/nrendo.2016.105

11. Malin SK, Kashyap SR. 2016. Type 2 diabetes treatment in the patient with obesity. Endocrinol Metab Clin North Am 45(3): 553-564. doi: 10.1016/j.ecl.2016.04.007

12. Jung UJ, Choi MS. 2014. Obesity and its metabolic complications: the role of adipokines and the relationship between obesity, inflammation, insulin resistance, dyslipidemia and nonalcoholic fatty liver disease. Int JMol Sci 15(4): 6184-6223. doi: 10.3390/ijms15046184

13. Cornell S. 2015. Continual evolution of type 2 diabetes: an update on pathophysiology and emerging treatment. Ther Clin Risk Manag 11: 621-632. doi: 10.2147/TCRM.S67387

14. Complications. [http://www.diabetes.org/living-with-diabetes/complications/]

15. Heart and Stroke Statistics. [http://www.heart.org/HEARTORG/ General/Heart-and-Stroke-Association-Statistics_UCM_319064_ SubHomePage.jsp]

16. Mandviwala T, Khalid U, Deswal A. 2016. Obesity and cardiovascular disease: a risk factor or a risk marker? Curr Atheroscler Rep 18(5): 21. doi: 10.1007/s11883-016-0575-4

17. Zezos P, Renner EL. 2014. Liver transplantation and non-alcoholic fatty liver disease. World J Gastroenterol 20(42): 15532-15538. doi: 10.3748/wjg.v20.i42.15532

18. Li L, Liu DW, Yan HY, Wang ZY, Zhao SH, et al. 2016. Obesity is an independent risk factor for non-alcoholic fatty liver disease: evidence from a meta-analysis of 21 cohort studies. Obes Rev 17(6): 510-519. doi: 10.1111/obr.12407 